\title{
Relation between age of mothers with breast cancer and sex of their children
}

\author{
H OLSSON, L BRANDT
}

\section{Summary and conclusions}

In a consecutive series of 150 women with breast cancer 122 had borne one or more children. Sixty-two patients were aged below 55 years at diagnosis (group $A$ ) and 60 were 55 years or older (group B). In group A 91 out of 153 children (59\%) were boys compared with 48 out of 141 $(34 \%)$ in group $B(p=0.000007)$. In group $A 54$ of the 62 patients $(87 \%)$ had given birth to one or more boys compared with 35 of the $60(58 \%)$ in group $B(p=0.0003)$. The mean age at diagnosis in mothers of two or more boys was 49.0 years, in those of one boy 55.2 years, and in those of only girls 61.0 years. The differences between each of the mean ages was significant. The mean age at diagnosis in 28 nulliparous patients was $\mathbf{5 7 . 7}$ years. There was no significant correlation between the number of female pregnancies and age at diagnosis.

These results suggest that in women liable to develop breast cancer male pregnancies are associated with an early onset of the disease.

\section{Introduction}

The reproductive history of premenopausal and postmenopausal women with breast cancer has been closely studied, ${ }^{1}$ but to our knowledge the sex distribution of their progeny has not been analysed. We have occasionally observed that the children of elderly women with breast cancer are predominantly girls. We have therefore compared the sex distributions of the children of young and old patients with breast cancer.

\footnotetext{
Department of Oncology, University Hospital, S-221 85 Lund, Sweden

H OLSSON, MD, resident physician

L BRANDT, MD, associate professor of internal medicine
}

\section{Patients and methods}

A consecutive series of 150 women with breast cancer treated at our department were interviewed about the sex and date of birth of each of their children. Stillbirths were also noted. The date of diagnosis of breast cancer was recorded, and the patients who had children were divided into two groups: those in whom breast cancer was diagnosed before the age of 55 (group A) and those in whom it was diagnosed at 55 years or over (group B). Statistical techniques used were Fisher's exact test and analysis of variance. All tests were two-sided.

\section{Results}

Sixty-two of the mothers were aged below 55 when breast cancer was diagnosed (group A) and 60 were 55 years or older (group B). The mothers in group A had 153 children-that is, 2.5 per woman-and those in group B 141 -that is, 2.4 per woman. Twenty-eight patients were nulliparous. Three stillbirths had occurred in group A and four in group B. In Group A the mean age at the first birth was 23.9 years and at the last birth 29.2 years. The corresponding ages in group B were $27 \cdot 1$ and $31 \cdot 8$ years respectively.

In group A 91 of the children were boys (59\%) compared with 48 in group B $(34 \%)$. 'This difference is significant $(\mathrm{p}=0.000007$, Fisher's exact probability test). In group A 54 of the 62 patients $(87 \%)$ had given birth to one or more boys compared with only 35 of the $60(58 \%)$ in group B $(\mathrm{p}=0.0003)$. Twenty-eight patients had given birth to only boys. Their mean age at diagnosis of breast cancer was 49.9 years. Thirty-three patients had given birth to only girls, and their mean age at diagnosis was 61.0 years (fig 1 ). The difference between the two groups with children of only one sex is significant $(p<0.0001)$. Sixty-one patients had both male and female children; their mean age at diagnosis was 53.2 years. This does not differ significantly from the mean age in the group with only boys but is significantly lower than that in the group with only girls $(\mathrm{p}<0.003)$.

Figure 2 shows the correlation between male pregnancies and an early onset of breast cancer. The mean age at diagnosis of breast cancer in mothers of two or more boys was 49.0 years and in those of one boy 55.2 years. The difference is significant $(p<0.02)$. In women with only girls the mean age at diagnosis was 61.9 for those with two or more girls and $60 \cdot 1$ for those with one. The mean age at diagnosis in the 28 nulliparous patients was $57 \cdot 7$ years (fig 1 ).

The data in the table show that the age at diagnosis of breast cancer becomes significantly lower as the number of boys in the offspring increases $\left(\mathrm{F}_{2,141}=1482 / 144=10 \cdot 28 ; \mathrm{p}<0.0001\right)$. The number of girls in the offspring has no significant influence $\left(F_{2,141}=208 / 144=\right.$ $1.44 ; \mathrm{p}>0.2$ ). 


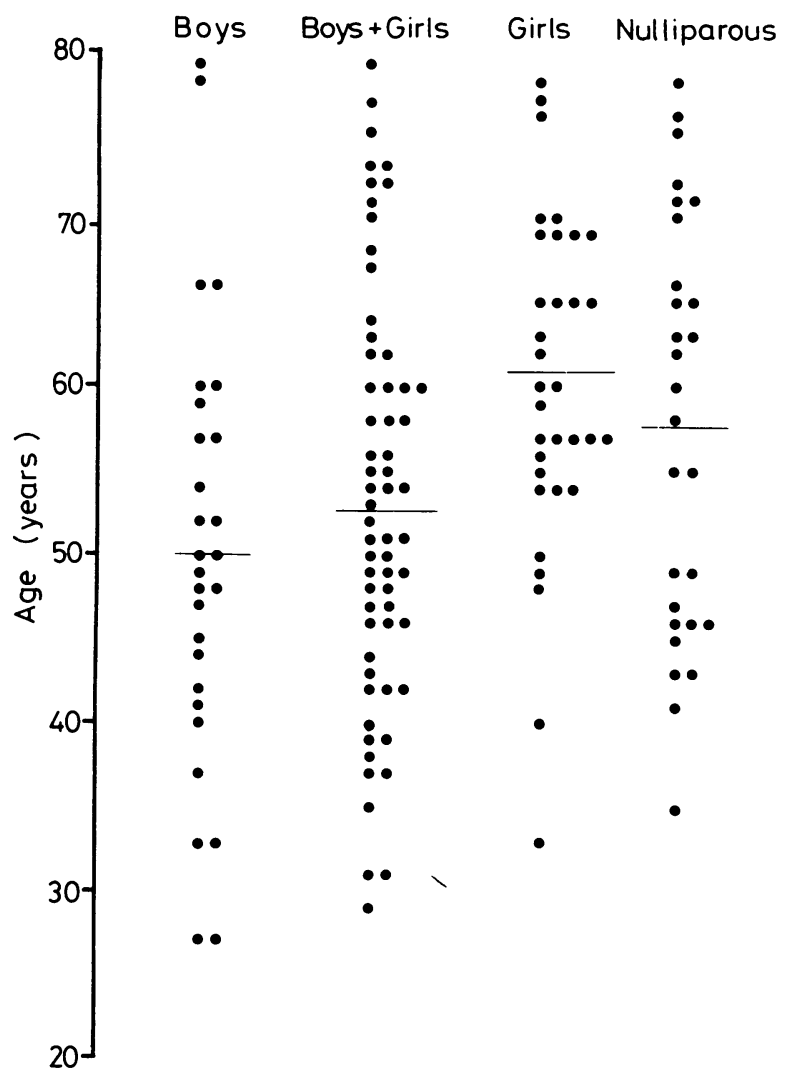

FIG 1-Age at diagnosis of breast cancer in mothers of only boys, boys and girls, and only girls. Ages of 28 nulliparous women are also shown. Mean values are indicated by bars.

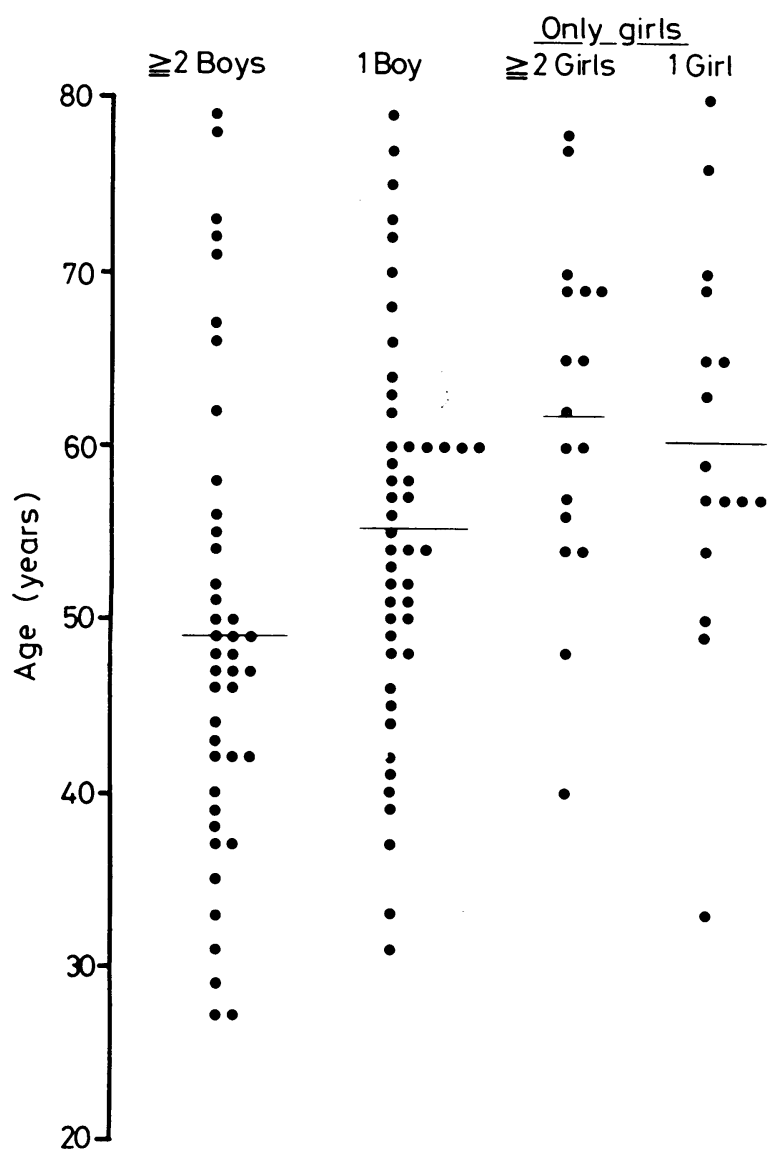

FIg 2-Relation between age at diagnosis and number of male pregnancies, and between age at diagnosis and number of female pregnancies in mothers of only girls.
Composition of sibships and mean $( \pm S D)$ age at diagnosis of breast cancer

\begin{tabular}{cccc}
\hline \multirow{2}{*}{$\begin{array}{c}\text { No of } \\
\text { girls }\end{array}$} & \multicolumn{3}{c}{ No of boys } \\
\cline { 2 - 4 } & 0 & 1 & 2 \\
\hline 0 & $57 \cdot 7 \div 12 \cdot 3$ & $51 \cdot 2=9 \cdot 3$ & $48 \cdot 7 ! 16 \cdot 9$ \\
$(\mathrm{n}=28)$ & $(\mathrm{n}=15)$ & $(\mathrm{n}=13)$ \\
1 & $60 \cdot 1=11 \cdot 3$ & $56 \cdot 4=10 \cdot 4$ & $48 \cdot 7 \pm 12 \cdot 1$ \\
$(\mathrm{n}=16)$ & $(\mathrm{n}=22)$ & $16)$ \\
2 & $61 \cdot 9 ! 10 \cdot 0$ & $58 \cdot 5+14 \cdot 5$ & $49 \cdot 5 \pm 11 \cdot 8$ \\
$(\mathrm{n}=17)$ & $(\mathrm{n}=10)$ & $(\mathrm{n}=13)$ \\
\hline
\end{tabular}

\section{Discussion}

Mothers in whom breast cancer developed at an early age had had more male pregnancies than mothers in whom breast cancer developed later, whereas a predominance of female pregnancies preceded the development of breast cancer in old age. The association between early onset of breast cancer and male pregnancies is confirmed by the finding of a mean age of only 49.9 years at diagnosis of breast cancer in mothers who had given birth to only boys. Moreover, the number of male pregnancies was related to the time of diagnosis of breast cancer. In mothers of two or more boys the mean age was 49.0 years, in those of one boy $55 \cdot 2$ years, and in those of only daughters 61 years.

The mean ages at the first and last births were lower in patients in group A than group B. Cancer was diagnosed in our patients in the late 1970s, so that the patients in group A were born after 1920 and most of those in group B before 1920. In Swedish women born in the years 1910-25 there was a continuous lowering of the reproductive ages. Thus in women born in 1910 the mean age at childbearing was 29.8 and in those born in 1925, 27.8 years. The ratio of boys to girls among the sibships did not change during this period." The differences in the reproductive ages between groups $\mathrm{A}$ and $\mathrm{B}$ were therefore to be expected, and the difference in sex distributions between the children of young and old patients with breast cancer is not due to a different sex ratio in the children of young and old women.

Renkonen ${ }^{3}$ suggested that the risk of getting cancer may be higher in mothers who have given birth to unisexual sibships consisting of more than two boys than in those of sibships of girls. ${ }^{3}$ In young patients with breast cancer whose first child is male a poorer prognosis has been reported than in those with a girl as the first child. ${ }^{4}$ Male pregnancies may therefore be of some importance for the incidence and prognosis of cancer in women. Our results suggest that male pregnancies may be associated with an early onset of breast cancer.

Results of epidemiological studies indicate that certain women form a high-risk group for breast cancer. ${ }^{5}$ According to the present results, women who belong to such a group and have given birth to boys may develop breast cancer earlier than those with only female children. Factors favouring the formation and development of male zygotes and other factors favouring an early onset of breast cancer may possibly operate in the women at risk. An alternative hypothesis is that male pregnancies may cause an early onset of breast cancer in women liable to develop the disease. The overrepresentation of girls born to patients who developed cancer in the postmenopausal period may then indicate that female pregnancies do not favour an early development of breast cancer. Aging may be a more important promoting factor in postmenopausal women belonging to a high-risk group. Such a hypothesis may be supported by the fact that nulliparous women who develop breast cancer are most often in the postmenopausal period $^{6}$ - that is, absence of male pregnancies is compatible with a late onset of breast cancer in women susceptible to the disease. In our group of nulliparous patients the mean age for development of breast cancer was almost as high as that in the mothers of only girls-namely, $57 \cdot 7$ years.

If male pregnancies do provoke an early onset of breast cancer in women at high risk for the disease the mechanisms behind such a causal relation remain unexplained. Hormonal differences between male and female pregnancies ${ }^{7}$ might be related to differences in the age at onset of breast cancer. Immunological 
mechanisms may also be relevant to the time of development of the disease. Possibly a greater or different type of maternal immune tolerance is required during a male compared with a female pregnancy. ${ }^{4}$ An increased immune tolerance during male pregnancies might then further the establishment and development of breast cancer in women liable to the disease.

In our study breast cancer was diagnosed before the age of 50 in 26 of the 42 women $\left(62^{\circ}\right.$ o $)$ who had given birth to more than one boy (fig 2). The corresponding figures for mothers of one boy were 13 out of $47\left(28^{\circ} \%\right)$ and for those of only girls four out of $33(12 \%)$. In most mothers of two or more boys screening for breast cancer over the age of 50 may therefore be too late, whereas in mothers of only girls early screening procedures may be less important. Information on the composition of the progeny in a larger group of patients than the present might therefore have some bearing on the optimal age for the start of screening procedures.

We are indebted to B Gullberg, tumour registry for southern Sweden, for valuable statistical advice.
Requests for reprints should be addressed to $\mathrm{H}$ Olsson, Department of Oncology, University Hospital, S-221 85 Lund, Sweden.

\section{References}

${ }^{1}$ Adami HO, Rimsten A, Stenkvist B, Vegelius J. Reproductive history and risk of breast cancer. A case-control study in an unselected Swedish population. Cancer 1978;41:747-57.

2 Bernhardt E. Trends and variations in Swedish fertility. A cohort study. Stockholm: Central Bureau of Statistics, 1971.

${ }^{3}$ Renkonen KO. Malignant tumours and the host's reactivity. Annales Medicinae Experimentalis et Biologiae Fenniae 1965;43:155-8.

${ }^{4}$ Janerich DT. Pregnancy, breast-cancer risk, and maternal-fetal genetics. Lancet 1979 ; $\mathrm{i}: 327-8$.

${ }^{5}$ Donegan WL. Epidemiology. In: Donegan WL, Spratt JS, eds. Cancer of the breast. 2nd ed. Philadelphia: W B Saunders, 1979:14-34.

${ }^{6}$ MacMahon B, Feinleib M. Breast cancer in relation to nursing and menopausal history. F Natl Cancer Inst 1960;24:733-53.

${ }^{7}$ Reyes FI, Boroditsky RS, Winter JSD, Fairman C. Studies on human sexual development. II. Fetal and maternal serum gonadotropin and sex steroid concentrations. F Clin Endocrinol Metab 1974;38:612-7.

(Accepted 1 September 1980)

\section{Summary and conclusions}

Prostaglandin $\mathrm{E}_{1}$, a vasodilator and potent inhibitor of platelet aggregation, was administered to 26 patients with severe vasospastic disease of the hands. Patients tolerated infusions well and reported appreciable symptomatic improvement. Five of eight ischaemic ulcers healed in six weeks. Non-invasive studies of blood flow were used to observe haemodynamic changes during and after infusions. The Doppler-derived radial artery pulsatility index fell from $8.8 \pm 0.6$ to $4.6+0.5$ (mean $\perp$ SEM), indicating hand vasodilatation. This fall was maintained 24 hours after infusion $(5 \cdot 9 \pm 0.9)$, but the index had returned to normal values at two weeks. The amplitude of finger pulse volume recordings increased (5.6 $\pm 0 \cdot 7$ $\mathrm{mm}$ to $23.8+3.4 \mathrm{~mm}$ ) and was raised two and six weeks after infusion (13.5 $2 \cdot 1 \mathrm{~mm})$. Hand temperature measured by infrared radiometry also increased $(27.4 \pm$ $0.7^{\circ} \mathrm{C}$ to $31 \cdot 2 \pm 1.2^{\circ} \mathrm{C}$ ). Intensity of digital vasospasm induced by cold water challenge was not objectively affected by prostaglandin $E_{1}$ despite an increased finger temperature after infusion. Nevertheless, patients reported less frequent and severe attacks.

Prostaglandin $\mathrm{E}_{1}$ given by central venous infusion is a safe new vasoactive agent that can produce appreciable symptomatic improvement by increasing digital perfusion, which may last for several weeks after treatment. Further study will define its mode of action and its place in the management of peripheral vascular disease.

Bristol Royal Infirmary, Bristol BS2 8HW

P C CLIFFORD, FRCs, British Heart Foundation research fellow

M F R MARTIN, MRCP, senior registrar in rheumatology

E JANE SHEDDON, DCR, technician

$R$ N BAIRD, FRCs, consultant senior lecturer in surgery

P A DIEPPE, MRCP, consultant senior lecturer in rheumatology

St Bartholomew's Hospital, London

J D KIRBY, MRCP, consultant dermatologist

\section{Introduction}

Vascular disease in the hands and feet produces a wide range of ischaemic changes varying from transient attacks of vasospasm to ulceration and gangrene. ${ }^{1}$ Digital vasospasm, first described by Maurice Raynaud in $1862,{ }^{2}$ is now defined as episodic attacks of vasoconstriction of the small arteries in response to cold or emotional stimuli. ${ }^{3}$ They may occur alone (Raynaud's disease) or in association with various diseases including connective tissue disorders, arteriosclerosis obliterans (Buerger's disease), and atherosclerosis. The basic abnormality is probably in the peripheral vessels, ${ }^{45}$ but patients may also have reduced fibrinolytic activity, raised plasma fibrinogen concentration, and increased blood viscosity. ${ }^{6-8}$ Thus the typical symptoms and signs of Raynaud's phenomenon are secondary to the intermittent obstruction of digital vessels ${ }^{4}{ }^{9}$ arising from these rheological or vessel-wall abnormaitities.

Management of patients suffering from severe Raynaud's phenomenon with or without trophic skin changes remains controversial. Vasodilators, sympathectomy, plasma exchange, and fibrinolytic enhancement are used with varying effect. ${ }^{610-13}$ Recently it has been suggested that prostaglandins may have a role in the management of peripheral vascular disease. ${ }^{14-19} \mathrm{We}$ describe the use of intravenous prostaglandin $E_{1}$, a vasodilator and inhibitor of platelet aggregation, ${ }^{2021}$ to treat severe digital ischaemia of varying aetiology. We used non-invasive measurements of limb blood flow to quantify the clinical effects.

\section{Patients and methods}

We studied 26 patients presenting with severe Raynaud's phenomenon and ischaemic skin changes. Seventeen were women and nine men (age range 33-76 (mean 52) years). Eighteen had progressive systemic sclerosis, six small-vessel-dominant atherosclerosis, and two primary Raynaud's disease. Five patients had already undergone digital amputation, and in eight ischaemic ulceration of the fingers was present. Patients were admitted to hospital for five days, and informed consent was obtained for each infusion, which was given via a central venous cannula over 72 hours. A cooled $\left(4^{\circ} \mathrm{C}\right)$ solution 